\title{
STORM Imaging of Mitochondrial Dynamics using a
}

\section{Vicinal-Dithiol-Proteins-Targeted Probe}

Bingling Chen", a , Wanjun Gong", a , Zhigang Yang, , a, Wenhui Pan ${ }^{\mathrm{a}}$, Peter Verwilst ${ }^{\mathrm{b}}$, Jinwoo Shin ${ }^{\mathrm{b}}$, Wei Yan ${ }^{\mathrm{b}}$, Liwei Liu ${ }^{\mathrm{a}}$, Junle $\mathrm{Qu}^{*}$, a and Jong Seung Kimª, ${ }^{*}$,

${ }^{a}$ Key Laboratory of Optoelectronic Devices and Systems of Ministry of Education and Guangdong Province, College of Physics and Optoelectronic Engineering, Shenzhen University, Shenzhen 518060, China

${ }^{b}$ Chemistry Department, Korea University, Seoul 02841, Korea

${ }^{\#}$ These authors contributed equally to this work

* Corresponding emails: zhgyang@szu.edu.cn; jlqu@szu.edu.cn; jongskim@korea.ac.kr

Abstract: Stochastic optical reconstruction microscopy (STORM) is a promising method for the visualization of ultra-fine mitochondrial structures. However, this approach is limited to monitoring dynamic intracellular events owing to its low temporal resolution. We developed a new strategy to capture mitochondrial dynamics using a compressed sensing STORM algorithm following raw data pre-treatments by a noise-corrected principal component analysis and K-factor image factorization. Using STORM microscopy with a vicinal-dithiol-proteins targeting probe, visualizing mitochondrial dynamics was attainable with spatial and temporal resolutions of $45 \mathrm{~nm}$ and $0.8 \mathrm{~s}$, notably, dynamic mitochondrial tubulation retraction of $\sim 746 \mathrm{~nm}$ in $1.2 \mathrm{~s}$ was monitored. The labeled conjugate was observed as clusters (radii, $\sim 90 \mathrm{~nm}$ ) distributed on the outer mitochondrial membranes, not yet reported as far as we know. This strategy is promising for the quantitative analysis of intracellular behaviors below the optical diffraction limit.

Keywords: STORM; Mitochondrial Imaging; VDP Targeting; Cyanine; Live cell imaging

\section{Indroduction}

Mitochondria are characterized by highly interconnected networks and dynamic changes ${ }^{1}$. The formation and maintenance of mitochondrial networks are mediated by the fusion of individual mitochondria and junctions between tubules ${ }^{2}$. Dynamic changes in the fusion-fission balance and mitochondrial tubular network formation 
determine the overall morphology and biological behaviors of mitochondria. Furthermore, in a process referred to as dynamic tubulation, tubules stretch to fuse with the membranes of other mitochondria ${ }^{3}$. These dynamic events facilitate the maintenance of normal mitochondrial functions, such as the interchange of mitochondrial materials, DNA integrity, respiratory capacity, response to cellular stress, and apoptosis ${ }^{4}$. However, disruptions in mitochondrial dynamics lead to abnormal development and human diseases, including neurodegenerative disorders and cancers ${ }^{5}$. Therefore, investigations of mitochondrial dynamics are critical to enable a deeper understanding of diseases related to mitochondrial dysfunctions.

Fluorescence microscopy is widely used to investigate single mitochondria in live cells ${ }^{6}$. However, imaging mitochondrial substructures using conventional optical microscopy is limited by the optical diffraction limit. Since the emergence of super-resolution imaging, it has become feasible to visualize ultra-fine structures ${ }^{7}$. Stochastic optical reconstruction microscopy (STORM) has an outstanding imaging ability for biological objects below $200 \mathrm{~nm}$ in size ${ }^{8}$. Fluorescent probes composed of fluorophores (e.g., Alexa647) and antibodies are favored for STORM imaging in fixed cells ${ }^{9,10}$. However, live cell imaging by STORM remains challenging owing to the lack of appropriate probes and low temporal resolution. In particular, direct STORM has rarely been used to evaluate mitochondrial dynamics.

Smart fluorescent probes that selectively target mitochondrial membrane-bound proteins would facilitate the imaging of mitochondrial membrane structures using STORM. Mitochondria possess abundant vicinal dithiol proteins (VDPs) with two proximal cysteine residues in their primary structures; they play essential roles in maintaining mitochondrial redox homeostasis, antioxidant defense, and redox signaling. Fluorescent arsenicals contain a 1, 3, 2-dithiarsenolane moiety capable of selectively 
binding to VDPs by forming covalent bonds ${ }^{11}$. They can label mitochondrial VDPs in situ, providing a basis for the application of STORM to mitochondria in live cells ${ }^{12}$. However, STORM involves sparse lighting and dilute labeling with fluorescent probes during sample preparation, and thousands of frames are needed to reconstruct a superresolution image, resulting in poor temporal resolution ${ }^{13,14}$. Temporal resolution can be improved by reducing the number of frames. Compressed sensing STORM (CSSTORM) is an $\ell_{1}$-norm minimization-based multi-molecule localization algorithm and is more suitable for dense fluorescence than single-molecule fitting. It can substantially shorten the time required for signal acquisition by cutting the number of required frames, and the temporal resolution can be further improved by increasing the acquisition frame rate of the electron multiplying charge coupled device (EMCCD) ${ }^{15}$. However, the rapid signal acquisition will increase background noise, thereby increasing interference in image reconstruction. For the application of STORM to live cell mitochondrial dynamics, the development of smart probes targeting mitochondrial membrane VDPs with an improved reconstruction algorithm for noise suppression is a promising strategy.

In this work, we performed STORM imaging of mitochondrial dynamics in live cells, and carried out the quantitative analysis of the target under STORM super-resolution microscopy. A new fluorescent marker suitable for STORM imaging was developed to selectively label VDPs on the outer mitochondrial membrane by simple co-culturing with cells. Meanwhile, we employed a moderate illuminating laser power to obtain as much single-frame emitter localiztion as possible and to reduce photobleaching and phototoxicity. The temporal resolution of STORM imaging can be achieved by reducing the laser power and increasing the $\mathrm{CCD}$ frame rate. To avoid interference from background noises, a combination of noise-corrected principal component analysis 
(NC-PCA) and K-factor image factorization were developed to treat the raw data prior to reconstruction using the CSSTORM algorithm. The principle and implementation of the algorithms were further described in the Supplemental Information (Figure S14S17). Using this strategy, we achieved STORM imaging of mitochondrial dynamics including fusion, fission, and mitochondrial tubulation. Furthermore, the VDP distribution on the outer mitochondrial membrane was quantitatively evaluated by hierarchical clustering analysis under STORM imaging.

\section{Results and Discussion}

Cy5 fluorescent dye is an ideal photoblinking candidate for STORM microscopy and 1, 3, 2-dithiarsenolane is a useful unit for the covalent binding of VDPs. A piperazine spacer was used as the linker (Figure 1A). Fluorescent probe 1 was prepared according to previously described protocols ${ }^{16}$ (see Supporting Inforamtion for details). Prior to its application in cell imaging, the spectral properties of $\mathbf{1}$ were studied. First, probe $\mathbf{1}$ in ethanol showed absorption maxima at $649 \mathrm{~nm}$ with a molar extinction coefficient of 2.1 $\pm 0.3 \times 10^{5}$ and emission maxima at $670 \mathrm{~nm}$, respectively (Figure $1 \mathrm{~B}$ ). The same parameters have also been tested in aqueous solution and similar results were obtained (Table S1 in Supplemental Information). A fluorescence quantum yield of 0.28 in ethanol and 0.18 in aqueous solution was obtained compared to Rhodamine B. Additionally, the photoblinking behavior of a single molecule of $\mathbf{1}$ was investigated using the home-built dSTORM system. In the absence of an imaging buffer and thiols ${ }^{7,17}$, a specimen of $\mathbf{1}$ was directly illuminated by a laser beam $\left(656 \mathrm{~nm}, 0.8 \mathrm{~kW} \cdot \mathrm{cm}^{-}\right.$ $\left.{ }^{2}\right)$ to show marked photoblinking fluorescence with a high photon number (ca. 3000 photons per pixel at each frame of the fluorescent state in the "on" cycle) and a low onoff duty cycle (ca. 0.0013). The mechanism of photoblinking under laser illumination 
was further discussed in the Supplemental Information (Supplementary Scheme 2). The duration of photoblinking lasts for more than 900 seconds with a suitable switching frequency, while the average photoblinking cycle of "on" state was approximately 88.2 ms (Figure 1C-F and Figure S1).

To evaluate the analytical results of the CSSTORM reconstruction after NC-PCA denoising and $\mathrm{K}$-factor sharpening, a fluorescent probe was placed on a coverslip to produce photoblinking under laser illumination at $656 \mathrm{~nm}$. The blinking dataset was analyzed by NC-PCA using different major principal components, as shown in Figure S17. Comparisons of 1-3 to 1-6 major principal components showed that the fewer principal components chosen for signal reconstruction, the more evident the noise reduction and the higher the potential for signal distortion. 1-6 major principal components were empirically employed to balance noise reduction and the fidelity of the signal in the NC-PCA de-noising process for super-resolution image reconstruction, based on the validation experiments of the NC-PCA algorithm by using simulation data (Figure S15-S16). When implementing the K-factor algorithm, the parameters $\mathrm{M}=54$ (number of factors) and $\mathrm{h}=9$ (number of harmonics) were set manually, and the k value was set automatically, gradually reducing according to the number of factors. The detailed workflow is presented in Figure S14. 
(A)

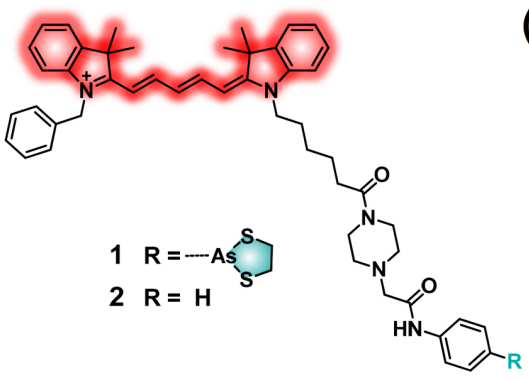

(C)

(D)

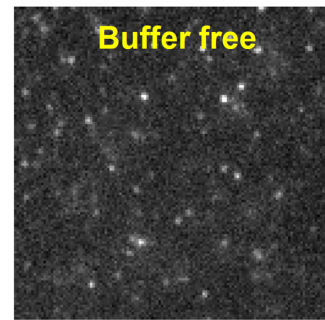

(B)
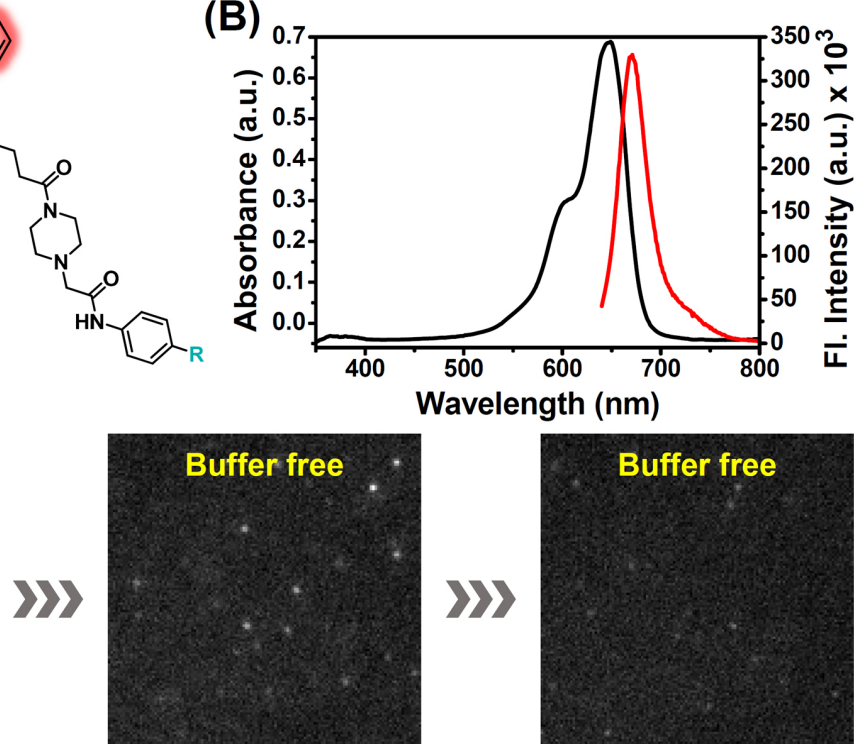

30 second
Buffer free
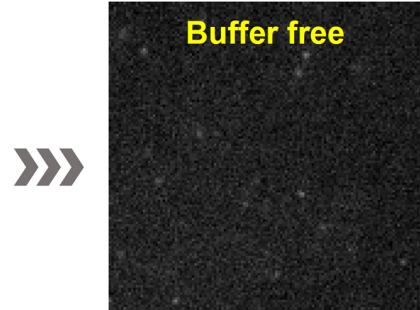

60 second

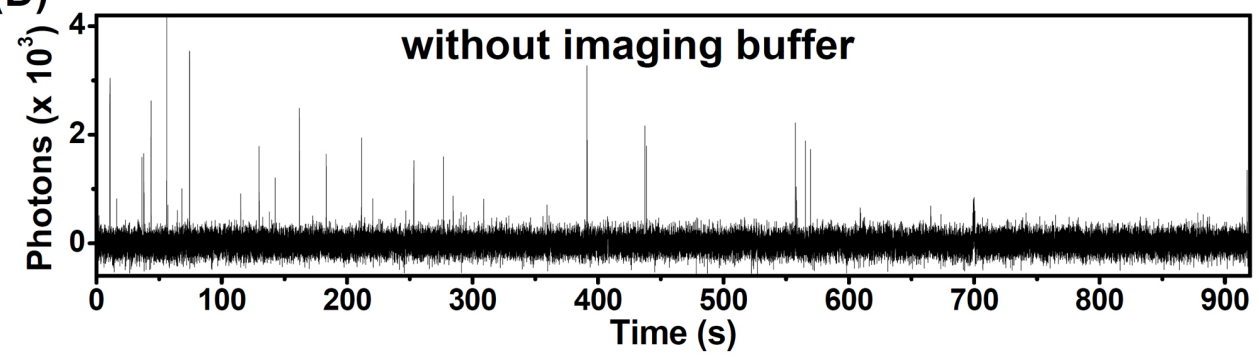

(E)

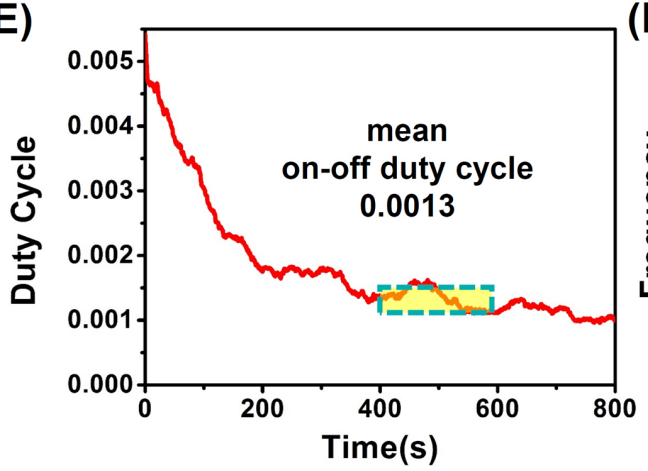

(F)

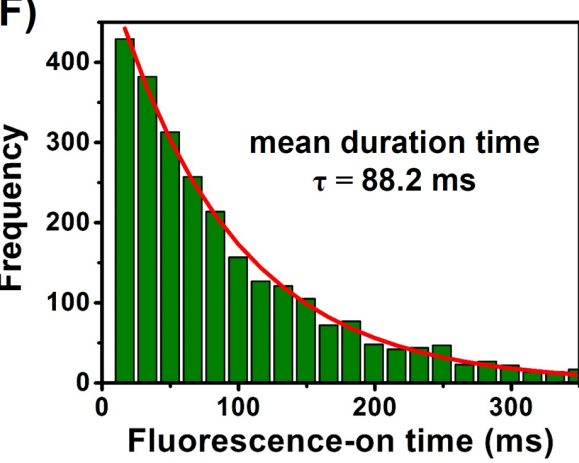

Figure 1. Chemical structures and optical properties of target compound 1 without imaging buffer. (A) Chemical structure of target compound 1; (B) Absorption (black) and emission (red) spectra of $\mathbf{1}$ in ethanol solution; (C) Reversible and spontaneous fluorescence blinking over tens of seconds in the absence of a STORM imaging buffer containing $\beta$-mercaptoethanol (BME) and glucose oxidase (GLOX), excited at $656 \mathrm{~nm}$ with a laser power of $0.8 \mathrm{~kW} \cdot \mathrm{cm}^{-2}$; (D) Time traces of single-molecule fluorescence of probe 1; (E) The on-off duty cycle value was calculated as the fraction of time spent in the on state averaged over many molecules using a sliding window of 100 seconds, the 
mean duty cycle was measured between 400-600 seconds indicated by yellow box; (F) Distribution of fluorescence-on time of probe 1, the indicated mean duration time of $88.2 \mathrm{~ms}$ was derived from the single exponential fit (red curve) of the histogram.

To further verify de-noising by $\mathrm{NC}-\mathrm{PCA}+\mathrm{K}$-factor treatment prior to CSSTORM localization, we compared the reconstruction results obtained by CSSTORM with those of the single-molecule fitting (insight3 software) algorithm, as shown in Figure 2, using tubulin with high-density labeling STORM data obtained from an openly available EPFL website ${ }^{18}$. The dataset was first de-noised using the NC-PCA algorithm and then sharpened using the K-factor algorithm. Similar structures of tubulins were obtained by CSSTORM (Figure 2A (a-c) and single-molecule fitting (Figure 2A (d)), and CSSTORM exhibited greater structural continuity using only 500 camera frames, and even as few as 200 frames; however, poor continuity was observed when using 100 frames, in a comparison between CSSTORM (Figure 2A (e-g, i-k, m-o)) and the insight 3 algorithm (Figure 2A $(\mathrm{h}, 1, \mathrm{p}))$. The magnified view in Figure 2A (a-d) shows markedly sharpened structures upon de-noising using the NC-PCA+K-factor algorithm (Figure 2A (g)), which maintained the most structural consistency with the insight3 results (Figure 2A (h)). Seven microtubules were distinguishable after NC-PCA+Kfactor treatment, and ambiguous noisy peaks inevitably appeared between adjacent microtubules in CSSTORM treatment of raw data or for K-factor treatment only, as shown by the dashed circles in Figure $2 \mathrm{~B}(\mathrm{a}-\mathrm{b})$. Fourier ring correlation (FRC) is a quantitative imaging-resolution measuring tool that has been used to further evaluate improvements in image resolution ${ }^{19}$. Image resolution $(\mathrm{R})$ was determined by the inverse of the spatial frequency indicated by the intersection of the FRC curve with a given threshold, which was empirically set to $1 / 7$ (Figure S18). NC-PCA+K-factor 
treatment resulted in the highest imaging resolution of $39 \mathrm{~nm}$, which was comparable to those obtained by CSSTORM for raw data $(66 \mathrm{~nm})$ and K-factor only $(62 \mathrm{~nm})$.
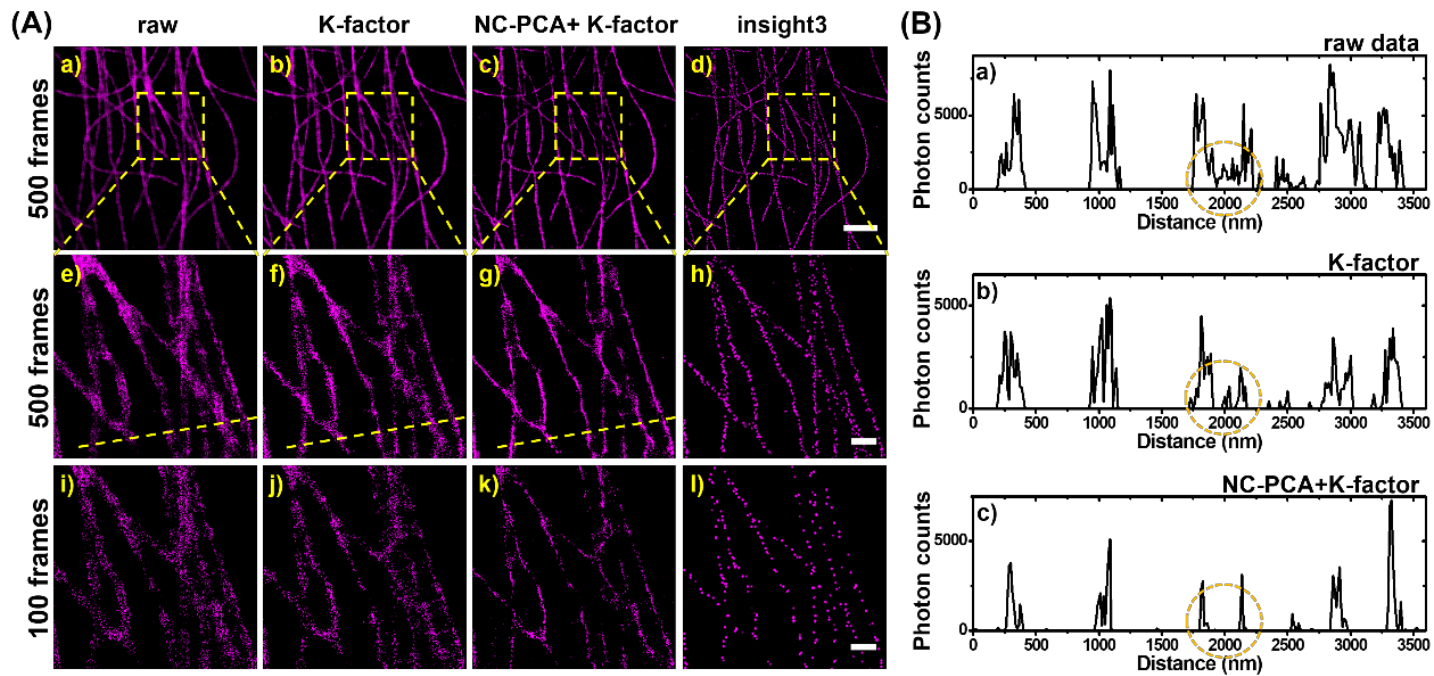

Figure 2. Validation of the NC-PCA de-noising algorithm using an experimental dataset of tubulins with high-density labeling (from EPFL). (A) Comparison of CSSTORM results for raw data (column 1), K-factor (column 2), and NC-PCA+Kfactor pre-treatment (column 3) and single-molecule fitting results (column 4). Row 24, magnified view of row 1, reconstructed using 500, 200 and 100 frames, respectively. Scale bar, $2 \mu \mathrm{m}$ in (d) and $500 \mathrm{~nm}$ in (h, 1, p); (B) Profiles along the yellow dashed lines shown in (e-g).

The biological behavior of probe $\mathbf{1}$, including colocalization, covalent binding identification, and cytotoxicity, was studied. The results demonstrated that probe $\mathbf{1}$ preferentially targeted mitochondria, demonstrated through the optimal merging imaging with Mito-tracker over Lyso-, ER-trackers (Figure S2A, S3 and S4). Moreover, mitochondrial VDPs were covalently labeled by the probe, which was identified by SDS-PAGE and washing-out experiments. The probe cannot be removed from mitochondria after washing with PBS, PBS-ethanol, or ethanol solvents, (Figure S2B, S2C, S2D, S5 and S6). Furthermore, the probe showed no cellular toxicity under our 
experimental conditions (Figure S7), which is favorable for live cell super-resolution imaging (Figure 3, S8).

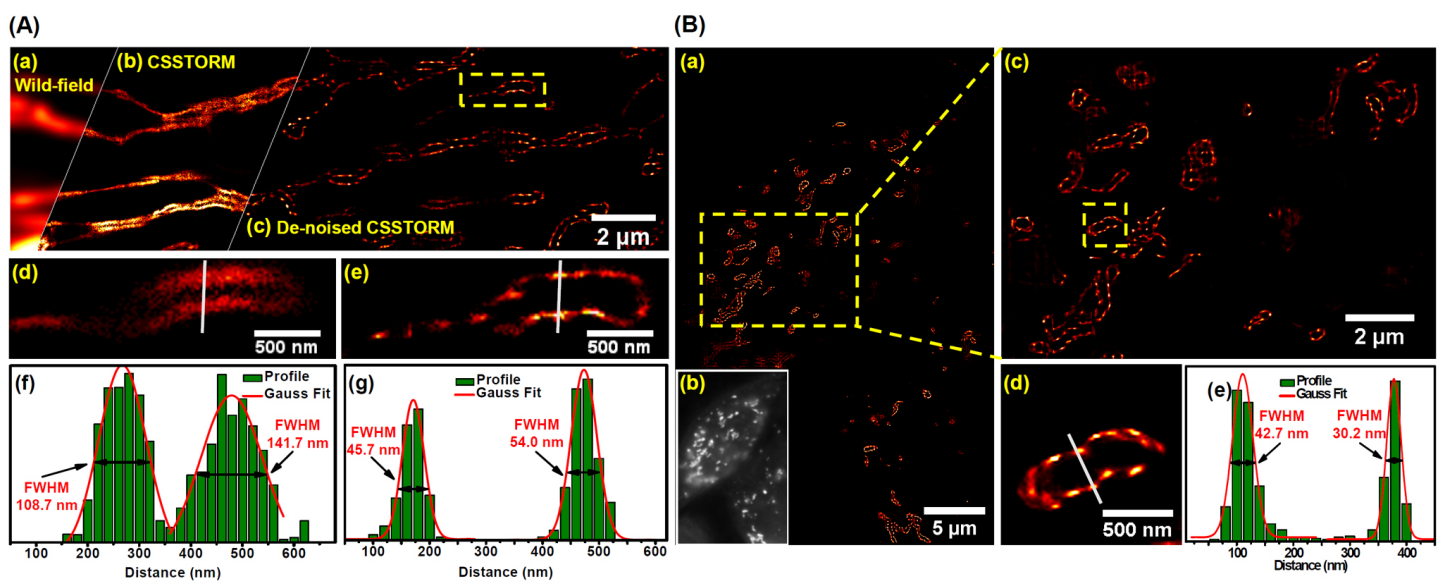

Figure 3. Super-resolution imaging of mitochondrial membranes labelled with probe $\mathbf{1}$ in live HeLa cells (A) and in fixed HeLa cells (B). (A) Wide-field fluorescent image (a), and STORM images (b and c) obtained by CSSTORM and de-noised CSSTORM algorithms; (d, e) magnified images of single mitochondria in panel c by CSSTORM and de-noised CSSTORM, respectively; (f, $g$ in A and e in B) Width analysis of the outer membrane; the blue histogram shows the cross-sectional profile along the lines in (d, e) in panel A and (d) in panel B; (B) Wide-field imaging (b); (a, c, d) STORM images obtained by the CSSTORM using 200 frames, EMCCD worked at a frequency of $250 \mathrm{~Hz}$ for $64 \times 128$ pixels raw data acquisition.

To identify the exact location of $\mathbf{1}$ in mitochondria of live cells, STORM superresolution imaging was performed to determine the ultra-fine structure of mitochondria labeled by 1 . Probe $1(1 \mu \mathrm{M})$ was incubated with HeLa cells for $1 \mathrm{~h}$ without further treatment and importantly this procedure was simpler than that using Alexa647. Subsequently, the cell samples were fixed with glutaraldehyde before imaging or used for live cell imaging by the STORM system. As shown in Figure 3A (a), unlike the wide-field image, which cannot provide the fine structure of mitochondria, the super- 
resolution images showed detailed structures, which were reconstructed using 200 frames by CSSTORM (Figure 3A (b)) and de-noised CSSTORM in live HeLa cells (Figure 3A (c)) or in fixed cells (Figure 3B). From the magnified image of the region of interest, indicated by dashed square in Figure 3A (c), the de-noised CSSTORM resolution was evidently higher than that of CSSTORM (Figure 3A (d)), and the discrete granule distribution on the mitochondrial membrane was clearly observed in Figure 3A (e), indicating that probe 1 selectively labeled VDPs on the mitochondrial membrane. The imaging resolutions of CSSTORM and de-noised CSSTORM were calculated by Gaussian fitting, showing full widths at half maximum (FWHM) of 108.7 and $45.7 \mathrm{~nm}$, respectively, as shown in Figure 3A (f, g). The covalent binding of 1 to VDPs on mitochondrial membranes was further supported by STORM using fixed HeLa cells. When HeLa cells were incubated with 1, fixed with glutaraldehyde and washed with PBS, similar super-resolution images of mitochondria were obtained by the de-noised CSSTORM algorithm (Figure 3B (a, c, d)), and the high imaging resolution (30.2 $\mathrm{nm}$ in Figure 3B (e) enabled the study of the distribution of VDPs on the outer mitochondrial membrane as well as their dynamics (Figure S8).

STORM was further applied to evaluate dynamic mitochondrial changes, including mitochondrial fusion, fission, and tubulation. Videos demonstrating the dynamics were obtained by the CSSTORM or de-noised CSSTORM algorithm using 200 camera frames for reconstructions with a step length of 20 frames to obtain a temporal resolution of $0.8 \mathrm{~s}$. The video results clearly revealed a markedly better resolution of the de-noised CSSTORM images compared with those of wide-field images (Figure 4A) and CSSTORM images (Figure 4B). After pre-treatment by the NC-PCA+K-factor method, the de-noised CSSTORM analysis demonstrated obvious noise suppression (Video S2), and the ultra-fine imaging of VDPs on mitochondrial membranes was 
achieved (Figure 4C, Figure S8, and Video S2-S6). Moreover, typical dynamics of mitochondria can be observed to quantify speeds of mitochondrial fusion, fission, and tubulation, which probably reflects the mitochondrial status in biological activities ${ }^{20,21}$. Single mitochondrial tubulation and microtubule stretching were captured (dashed square in Figure 4C), and different time points are shown in Figure 4D (1-6). The distance during the tubulation retraction was $\sim 746 \mathrm{~nm}$, and finished in $1.2 \mathrm{~s}$ (Figure 4E). The hollow tube diameter (magnified dashed circle in Figure 4F), as calculated by Gaussian fitting, was $51.8 \mathrm{~nm}$, and FWHM values for membrane widths were $46.9 \mathrm{~nm}$ and $43.5 \mathrm{~nm}$ (Figure 4G), comparable to previously reported results ${ }^{22}$. Mitochondrial fusion and fission dynamics are shown in Figure S9 and Video S3-S8, which have never been achieved by traditional optical microscopy.

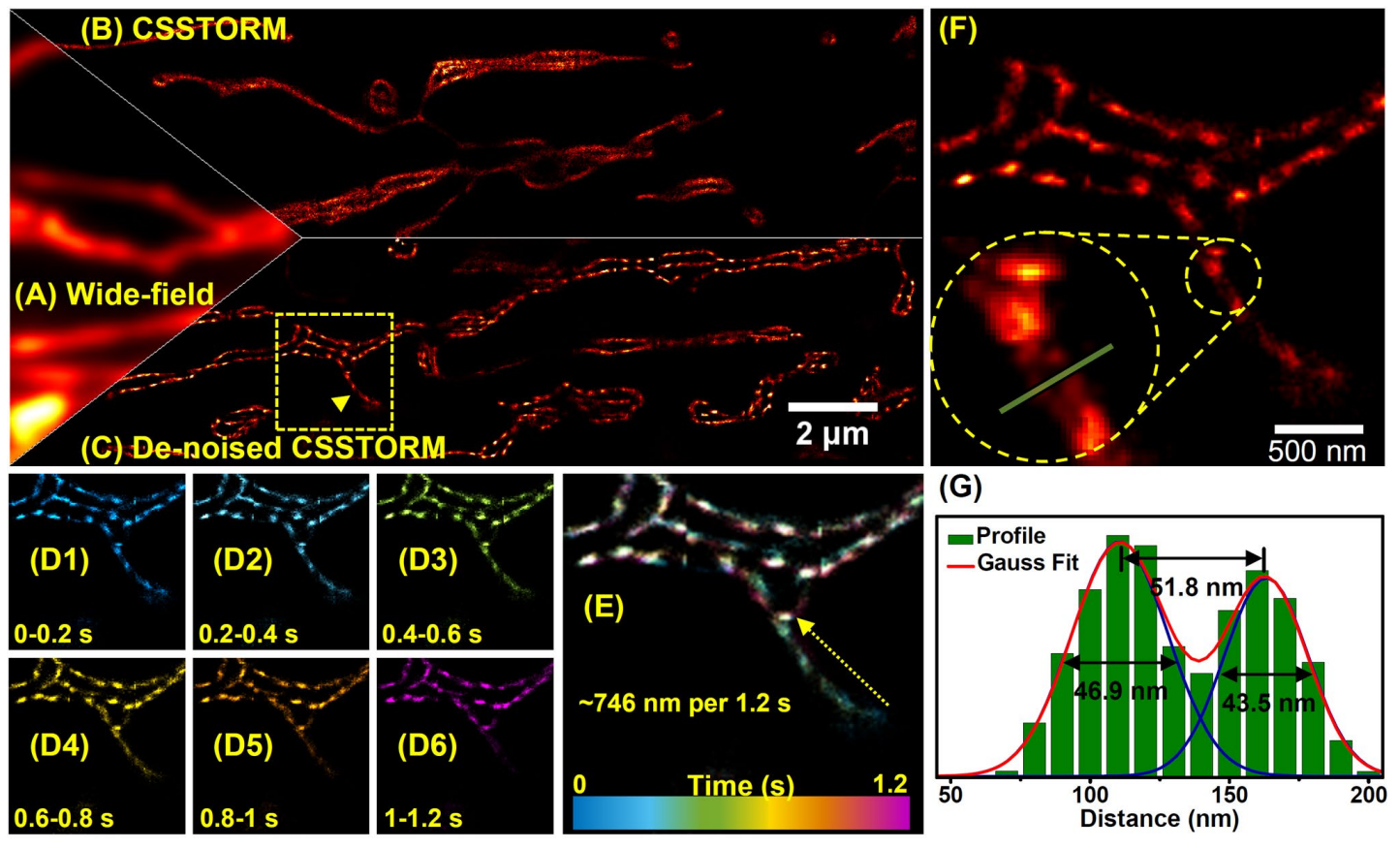

Figure 4. Mitochondrial tubulation analysis based on super-resolution imaging. (A) Wide-field image of mitochondria; $(\mathrm{B}, \mathrm{C})$ Super-resolution images of mitochondria in live HeLa cells analyzed by CSSTORM and de-noised CSSTORM algorithms; (D, E) Mitochondrial tubulation movement, (D1-D6) Different colours represent the movement status of mitochondria at different time points, (E) Whole tubulation 
retraction process by merging D1-D6; (F) Magnified single mitochondria and stretching tubule in (C); (G) Width analysis of mitochondrial tubules; blue histogram shows the cross-sectional profile along the line marked in the dashed circle in (F) with FWHM values obtained from Gaussian fitting (red and green).

Studies of the distribution of VDPs on mitochondrial membranes labeled by $\mathbf{1}$ can provide insight into biological functions. We used the open source software LocAlization Microscopy Analyzer (LAMA) ${ }^{23}$ to analyze morphological clusters of VDPs based on density-based hierarchical clustering algorithms, i.e., a density-based algorithm for discovering clusters in large spatial databases with noise (DBSCAN) and ordering points to identify the clustering structure (OPTICS). Considering the pixelation of the compressed sensing algorithm without directly returning a list of molecule coordinates, the CSSTORM results were first converted to a molecule list as input for LAMA by identifying clusters of nonzero grid points. A region of interest, a $128 \times 128$ pixels super-resolution grid image, was chosen for the protein clustering analysis (Figure 5A). The observation radius was set to $19 \mathrm{~nm}$, the minimum cluster size was set to 6 in DBSCAN, and the noise level was set to $13.1 \%$ in OPTICS $^{24}$. In total, 32 and 36 clusters were detected by DBSCAN and OPTICS, respectively, and are shown as 2D distributions of cluster populations in Figure 5 (B, E). A DBSCAN-based hierarchical cluster analysis was performed using the results of DBSCAN or OPTICS to extract morphological information for individual clusters by polygon formation. The morphological cluster analysis results with 16 false colors are summarized in Figure 5 (C, F). Finally, a cluster population distribution with a cluster size of $c a .90 \mathrm{~nm}$ was obtained, as shown in Figure $5(\mathrm{D}, \mathrm{G})$, indicating that this is a promising strategy for analyses of protein distributions in live cells (Figure S10-S13). 

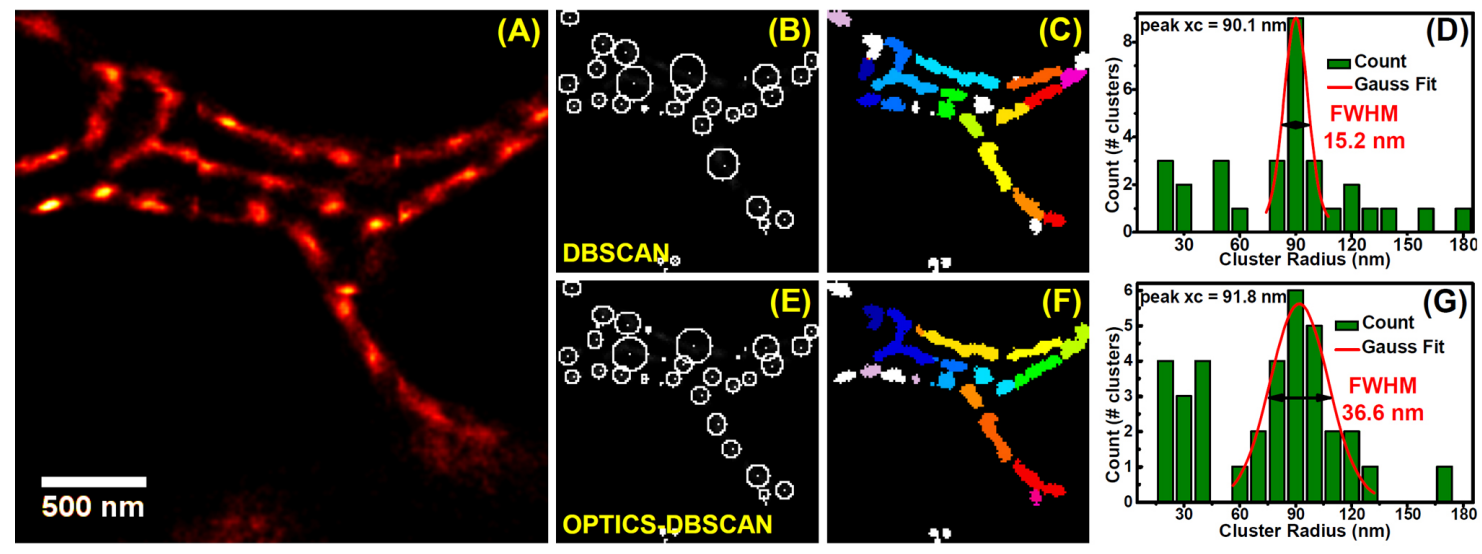

Figure 5. Distribution of mitochondrial membrane VDPs determined by a protein clustering analysis-using DBSCAN (B, C, D) and OPTICS (E, F, G). (A) STORM super-resolution image (scale bar $500 \mathrm{~nm}$ ); (B, E) 2D distribution of the cluster population; (C, F) Visual representation of the DBSCAN-based morphological cluster analysis; (D, G) Distribution of cluster radii.

\section{Conclusion}

In conclusion, a promising strategy for STORM super-resolution microscopy was developed to carry out mitochondrial dynamics imaging in live cells. In this method, a new STORM probe was applied to covalently bind to VDPs in the mitochondrial outer membrane, which achieved STORM super-resolution imaging without the need for an imaging buffer and thiols. The mitochondrial dynamics analyses were obtained by CSSTORM imaging using a noise-correction method consisting of a NC-PCA $+\mathrm{K}-$ factor pre-treatment. De-noising CSSTORM enabled ultra-fine structural imaging of mitochondria with a spatial resolution of up to $30.2 \mathrm{~nm}$ in fixed cells and 45.7 in live cells with a temporal resolution of $0.8 \mathrm{~s}$. The dynamic changes of the 1-VDP complex on the mitochondrial membrane were utilized to demonstrate mitochondrial dynamics, including fusion, fission, and tubulation, in particular, dynamic tubulation retraction of ca. $746 \mathrm{~nm}$ in $1.2 \mathrm{~s}$. The distribution of VDPs on the outer mitochondrial membrane 
was further analyzed as protein aggregation $(c a .90 \mathrm{~nm})$ to gain a deeper insight into their biological functions. The newly developed strategy will be useful for further functional studies of mitochondria.

\section{Acknowledgements}

This work was supported by the National Natural Science Foundation of China $(61875131 / 61525503 / 61620106016 / 61835009)$ and Key Project of Department of Education of Guangdong Province (2015KGJHZ002/2016KCXTD007). This work was supported by the National Research Foundation of Korea (NRF) funded by the Ministry of Science and ICT (CRI project no. 2018R1A3B1052702, JSK)

\section{Reference}

1. J. Nunnari, A. Suomalainen, Cell 2012, 148, 1145-1159.

2. S. Meeusen, J. M. McCaffery, J. Nunnari, Science 2004, 305, 1747-1752.

3. C. Wang, W. Du, Q. P. Su, M. Zhu, P. Feng, Y. Li, Y. Zhou, N. Mi, Y. Zhu, D. Jiang, S. Zhang, Z. Zhang, Y. Sun, L. Yu, Cell Res. 2015, 25, 1108-1120.

4. D. C. Chan, Annu. Rev. Genet. 2012, 46, 265-287.

5. H. Chen, D. C. Chan, Hum. Mol. Genet. 2009, 18, 169-176.

6. H. Zhu, J. Fan, J. Du, X. Peng, Acc. Chem. Res. 2016, 49, 2115-2126.

7. a) S. A. Jones, S. H. Shim, J. He, X. Zhuang, Nat. Methods 2011, 8, 499-508; b) S.-N. Uno, M. Kamiya, T. Yoshihara, K. Sugawara, K. Okabe, M. C. Tarhan, H. Fujita, T. Funatsu, Y. Okada, S. Tobita, Y. Urano. Nat. Chem. 2014, 6, 681-689; c) A. Small, S. Stahlheber. Nat. Methods, 2014, 11, 267-279; d) S. Alexey, R. M. Hochstrasser. Proc. Natl. Acad. Sci. U. S. A. 2006, 103, 18911-18916; e) R. Iinuma, Y. Ke, R. Jungmann, T. Schlichthaerle, J. B. Woehrstein, P. Yin. Science, 2014, 
$344,65-69$.

8. Y. Doksani, J. Y. Wu, T. de Lange, X. Zhuang, Cell 2013, 155, 345-356.

9. a) M. Bates, B. Huang, G. T. Dempsey, X. Zhuang, Science 2007, 317, 17491753; b) B. Huang, W. Wang, M. Bates, X. Zhuang, Science 2008, 319, 810-813.

10. S. Shen, X-F. Li, W. R. Cullen, M. Weinfeld, X. C. Le, Chem. Rev. 2013, 113, 7769-7792.

11. C. Huang, Q. Yin, W. Zhu, Y. Yang, X. Wang, X. Qian, Y. Xu, Angew. Chem. Int. Ed. 2011, 50, 7551-7556.

12. a) L. Zhu, W. Zhang, D. Elnatan, B. Huang, Nat. Methods 2012, 9, 721-723; b) J. Huang, K. Gumpper, Y. Chi, M. Sun, J. Ma, Opt. Lett. 2015, 40, 2989-2992.

13. S. H. Shim, C. Xia, G. Zhong, H. P. Babcock, J. C. Vaughan, B. Huang, X. Wang, C. Xu, G. Q. Bi, X. Zhuang, Proc. Natl. Acad. Sci. U.S.A. 2012, 109, $13978-13983$

14. X. Peng, Z. Yang, J. Wang, J. Fan, Y. He, F. Song, B. Wang, S. Sun, J. Qu, J. Qi, M. Yan, J. Am. Chem. Soc. 2011, 133, 6626-6635.

15. S. N. Uno, M. Kamiya, T. Yoshihara, K. Sugawara, K. Okabe, M. C. Tarhan, H. Fujita, T. Funatsu, Y. Okada, S. Tobita, Y. Urano, Nat. Chem. 2014, 6, 681-689.

16. http://bigwww.epfl.ch/smlm/datasets/.

17. R. P. Nieuwenhuizen, K. A. Lidke, M. Bates, D. L. Puig, D. Grunwald, S. Stallinga, B. Rieger, Nat. Methods 2013, 10, 557-562.

18. a) P. Mishra, D. C. Chan, J. Cell Biol. 2016, 212, 379-387; b) D. Sebastian, M Palacin, A. Zorzano, Trends Mol. Med. 2017, 23, 201-215.

19. M. Lavorato, V. R. Iyer, W. Dewight, R. R. Cupo, V. Debattisti, L. Gomez, S. De la Fuente, Y-T. Zhao, H. H. Valdivia, G. Hajnoczky, C. Franzini-Armstrong, Proc. Natl. Acad. Sci. U. S. A. 2017, 114, 849-858. 
20. P. R. Nicovich, D. M. Owen, K. Gaus, Nat. Protoc. 2017, 12, 453-460.

21. S. Jahirabadkar, P. Kulkarni, Expert Syst. Appl. 2014, 41, 2939-2946.

22. Y. Wang, S. Jia, H. F. Zhang, D. Kim, H. Babcock, X. Zhuang, L. Ying, Optica $2017,4,1277-1284$.

23. P. R. Nicovich, D. M. Owen, K. Gaus, Nat. Protoc. 2017, 12, 453-460.

24. S. J. Holden, S. Uphoff, A. N. Kapanidis, Nat. Methods 2011, 8, 279-280. 


\section{Graphic Abstract}

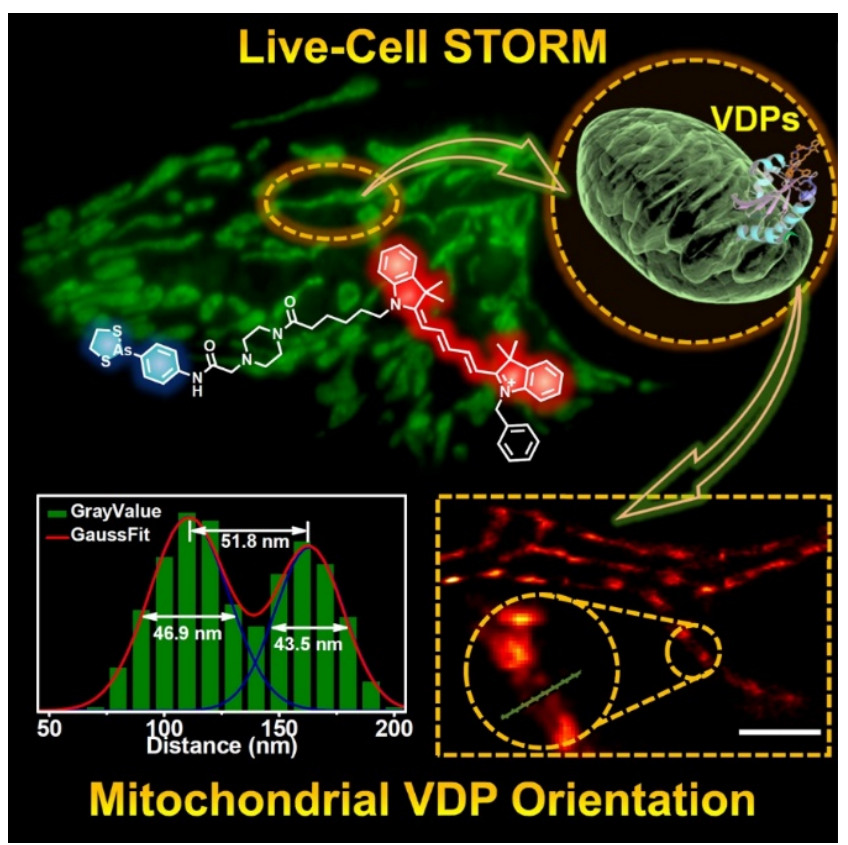

A new STORM probe was developed to selectively label vicinal dithiol proteins on the outer mitochondrial membrane, achieved STORM imaging of mitochondrial dynamics in live cells. By pre-treating with a noise-correction-principal-component-analysis and a K-factorization algorithm, a compressed sensing algorithm achieved STORM imaging with enhanced temporal resolution. Mitochondrial dynamics, were visualized with ultra-fine details to facilitate the studies on mitochondrial functions in live cells at resolutions beyond the optical diffraction limit. 